\title{
ADSORÇÃO EM MEIO AQUOSO DO CORANTE AZO PONCEAU 4R SOBRE SÍLICA GEL ORGANOFUNCIONALIZADA COM APTES
}

\author{
Alessandra Vieira de Lima ${ }^{1}$, Pérola Aryel Lima Mota ${ }^{1}$, Ana Flávia dos Santos ${ }^{1}$, \\ Nayane Gonçalves Nogueira ${ }^{1}$, Aline de Araújo Viana ${ }^{1}$, Rinaldo dos Santos Araújo ${ }^{1}$, \\ Francisco Murilo Tavares de LunA ${ }^{2}$, Hugo Leonardo de Brito Buarque ${ }^{1}$ \\ ${ }^{1}$ Instituto Federal de Educação, Ciência e Tecnologia do Ceará (IFCE) - Campus Fortaleza \\ ${ }^{2}$ Universidade Federal do Ceará (UFC) \\ <ale.vieiralimaa@gmail.com.br>, <perolalmota@gmail.com.br> \\ <aninha_flavia_santos@hotmail.com>, <nayane_gn@ hotmail.com>, <viana_aline@yahoo.com.br> \\ <rinaldo@ifce.edu.br>,<murilo@gpsa.ufc.br>,<hbuarque@ifce.edu.br>
}

DOI: 10.21439/conexoes.v10i2.775

Resumo. Os corantes azoicos constituem a classe de corante mais usada industrialmente, constituindo alguns dos principais contaminantes de efluentes industriais. O corante Ponceau 4R (Acid Red 18) é um corante azoico utilizado em diversas indústrias têxteis e alimentícias e deve ser eficientemente removido de águas residuais industriais. Neste estudo, avaliou-se a utilização de sílica gel comercial modificada superficialmente com 3-aminopropiltrietoxisilano (APTES) na remoção do corante Ponceau 4R. Preliminarmente, foram realizadas a funcionalização da sílica gel com APTES e a otimização do $\mathrm{pH}$ dos ensaios adsortivos. Então, dados de cinética e de equilíbrio de adsorção foram determinados, em temperatura de $30{ }^{\circ} \mathrm{C}$, pH 3,0 e agitação de $160 \mathrm{rpm}$. Modelos cinéticos de pseudo-primeira ordem e pseudo-segunda ordem, e isotermas de equilíbrio de Langmuir e de Freundlich, também foram ajustados aos dados determinados. Capacidades de remoção do azo-composto superiores a $47 \mathrm{mg} / \mathrm{g}$ foram obtidos com velocidades de adsorção relativamente elevadas, indicando que o adsorvente avaliado é bastante promissor na remoção do corante azoico estudado. Mencione-se, ainda, que o modelo de pseudo-segunda ordem se ajustou bem aos dados cinéticos, enquanto que ambas as isotermas de Langmuir e Freundlich se mostraram insatisfatórias na representação dos dados de equilíbrio determinados.

Palavras-chaves: Corantes azoicos. Acid Red 18. Sílica gel modificada. 3-aminopropiltrietoxisilano.

\begin{abstract}
Azo dyes are the class of dyes most used industrially, resulting some major contaminant of industrial effluents. Ponceau 4R (Acid Red 18) is an azo dye used in various food and textile industries, and must be efficiently removed from their wastewater. In this study, the removal of Ponceau $4 \mathrm{R}$ using commercial silica gel modified superficially with 3-aminopropyltriethoxysilane (APTES) has been evaluated. Preliminarily, it was confirmed the APTES functionalization on the commercial silica gel and it was carried out the $\mathrm{pH}$ optimization of the adsorption tests. So, kinetic data and equilibrium adsorption were determined at a temperature of $30{ }^{\circ} \mathrm{C}, \mathrm{pH} 3.0$ and agitation of $160 \mathrm{rpm}$. Models of pseudo-first order and pseudo-second order were fitted to kinetic data. Equilibrium data were applied to the common isotherm models namely the Freundlich and Langmuir. Adsorption capacity exceeding $47 \mathrm{mg} / \mathrm{g}$ were obtained with relatively high adsorption rates, indicating that the evaluated adsorbent is very promising in the removal of the azo dye studied. It is important to mention that pseudo-second order model fits well to the kinetic data, while both isotherms tested were unsatisfactory in representing equilibrium data determined
\end{abstract}

Keywords: Azo dyes. Acid Red 18. Modified silica gel. 3-aminopropyltriethoxysilane. 


\section{INTRODUÇÃO}

Diversas indústrias utilizam corantes em seus processos e produtos, tais como as indústrias têxteis e as alimentícias, produzindo águas residuais altamente coloridas, as quais, se não adequadamente tratadas, podem causar impactos deletérios nos corpos aquáticos em que são lançadas (MALIK; RAMTEKE; WATE 2007; SHARMA et al. 2011; SURESH; SUGUMAR; MAIYALAGAN, 2011).

Os corantes do tipo azo constituem a classe de corante mais usada industrialmente, correspondendo a mais da metade dos corantes comercialmente utilizados (FAHMI ARIFFIN et al., 2010, FRANCISCON et al. 2012; NAM; RENGANATHAN; TRATNYEK, 2001, YIGITOGLU; TEMOCIN, 2010). Ressalte-se que estes compostos são de difícil remoção por tratamentos convencionais em efluentes industriais (e.g., lodo ativado, coagulação/floculação), a despeito de poderem se tornar tóxicos, carcinogênicos e mutagênicos no ambiente (GUPTA; SUHAS., 2009, KODAM; GAWAI, 2006, MAHMOODI et al., 2014; NAM; RENGANATHAN; TRATNYEK, 2001, PHAM; KOBAYASHI; ADACHI 2015; YIGITOGLU; TEMOCIN, 2010).

Desta forma, o tratamento de efluentes contendo corantes azoicos constitui um vasto e promissor campo de estudos (ABRAMIAN; EL-RASSY, 2011; BOUCHERIT; ABOUSEOUD; ADOUR, 2013; ZHANG et al. 2012). Ademais, os processos de adsorção têm se mostrado muito eficientes e economicamente viáveis no tratamento de efluentes contendo corantes azoicos (ABRAMIAN; EL-RASSY, 2011, AHMAD; HAMEED, 2010; CESTARI et al., 2009; GUPTA et al. 2011; ROY; ADHIKARI; MAJUMDER 2013; SHIRMARDI et al., 2012, WANG, 2012). As principais vantagens dos processos adsortivos no tratamento de efluentes coloridos são os menores investimentos relativos, simplicidade de projeto e operação, não suscetibilidade a compostos tóxicos gerados, e facilidade de recuperação do adsorvente, como também dos compostos adsorvidos (MAHMOODI et al., 2014, MITTAL et al. 2009, SHARMA et al., 2011, ZHANG et al., 2015).

Nos últimos anos, alguns estudos têm mostrado que adsorventes comerciais de baixo custo (e.g., sílica gel) modificados superficialmente podem ser eficientes na remoção de contaminantes ambientais (ANDRZEJEWSKA; KRYSZTAFKIEWICZ; JESIONOWSKI, 2007, CESTARI et al., 2009; DONIA et al. 2009, KRYSZTAFKIEWICZ; BINKOWSKI; JESIONOWSKI, 2002, KUSHWAHA; GUPTA; CHATTOPADHYAYA, 2010; MAHMOODI; KHORRAMFAR; NAJAFI, 2011; XIA et al., 2011; YIGITOGLU; TEMOCIN 2010). Assim, adsorventes silicosos superfici- almente organofuncionalizados com diferentes grupos funcionais ativos são de particular interesse. Estes materiais são capazes de interagir com diversas substâncias orgânicas quando enxertados com compostos quelantes, fazendo deles adsorventes promissores para a remoção seletiva de poluentes.

Neste contexto, objetivou-se nesse estudo avaliar as condições de utilização de uma sílica gel comercial modificada superficialmente com 3aminopropiltrietoxisilano (APTES) na adsorção em fase aquosa do corante Ponceau 4R (Acid Red 18), um azo-composto comumente usado em indústrias alimentícias, como também determinar dados fundamentais desse processo. Assim, a melhor faixa de $\mathrm{pH}$ para a adsorção do corante, bem como dados cinéticos e de equilíbrio foram determinados e modelados para soluções sintéticas do azo-composto na temperatura de $30^{\circ} \mathrm{C}$.

\section{MATERIAL E MÉTODOS}

\subsection{Soluções corantes}

Uma solução estoque com $2000 \mathrm{mg} / \mathrm{L}$ do corante aniônico Ponceau 4R (Merck, Alemanha), C.I. 16.255, cuja estrutura molecular está esquematizada na Figura 1 , foi utilizada na preparação de todas as soluções desse corante azoico utilizadas nesse estudo.<smiles>O=S(=O)([O-])c1cc(S(=O)(=O)[O-])c2c(N=Nc3ccc(S(=O)(=O)O[Na])c4ccccc34)c(O)ccc2c1</smiles>

Figura 1: Estrutura Molecular do Ponceau 4R (Acid Red 18).

A quantificação do corante em solução aquosa nos diversos ensaios executados foi conduzida em espectrofotômetro de absorção UV/VIS (Shimatzu UVPC 1601, Shimadzu Corp., Japan), calibrado para este fim no comprimento de onda de $510 \mathrm{~nm}$, após tamponamento a pH 7,0. As condições de calibração e quantificação das soluções de corante foram previamente determinadas e otimizadas a partir de varreduras espectrofotométricas realizadas em diferentes concentrações de corante e valores de $\mathrm{pH}$ (tampões). 


\subsection{Adsorvente}

Uma sílica gel branca (Vetec Química, Brasil), com granulometria de 1 a $4 \mathrm{~mm}$, foi utilizada na preparação da sílica gel modificada (SGM) utilizada como adsorvente nesse estudo. Previamente a essa preparação a sílica gel branca era macerada e peneirada, de modo que a granulometria das partículas diminuísse para valores entre 75 e $150 \mu \mathrm{m}$, de modo a melhorar a cinética do processo adsortivo.

A modificação da sílica gel consistia na sua funcionalização com APTES (Sigma-Aldrich, EUA), segundo o procedimento de Donia et al. (2009). A Figura 2 ilustra a estrutura molecular da superfície da SGM. A modificação da sílica gel foi confirmada, comparandose os espectros obtidos em espectrômetro infravermelho (Shimadzu IRAffinity-1, Shimadzu Corp., Japão), àqueles obtidos por Donia et al. (2009).

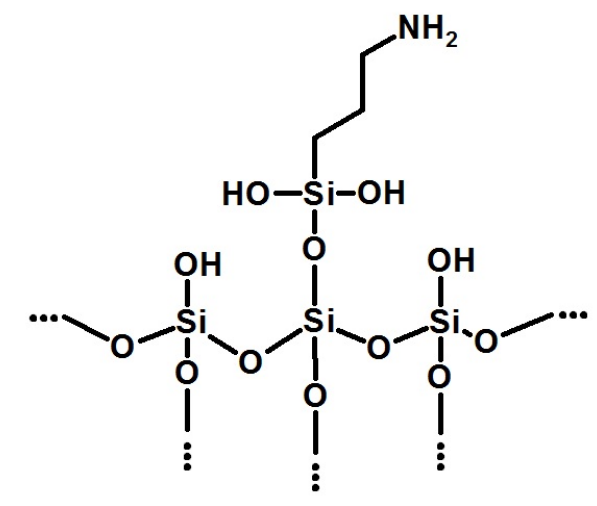

Figura 2: Estrutura Molecular da sílica gel modificada.

\subsection{Otimização do pH para os ensaios de adsorção}

Inicialmente, ensaios de ponto de carga zero (PCZ) foram realizados para a SGM, seguindo o procedimento de Guilarduci et al. (2006), de modo a estimar o pH de mudança da carga superficial do adsorvente. Posteriormente, foram avaliados por planejamento experimental os valores de $\mathrm{pH}$ ótimos para os ensaios de adsorção, numa faixa de $\mathrm{pH}$ de 3,0 a 9,0, e de concentração inicial do corante (Co) de 30 a $120 \mu \mathrm{mol} / \mathrm{L}(18 \mathrm{mg} / \mathrm{L}$ a 72 $\mathrm{mg} / \mathrm{L})$.

Nesse planejamento utilizou-se a técnica de delineamento composto central rotacional (DCCR) com quatro pontos axiais e quatro pontos centrais e da análise de curvas de superfícies para tal avaliação (RODRIGUES; EMMA, 2005). Os valores de pH e de concentração inicial do adsortivo (fatores) usados neste delineamento são mostrados na Tabela 1. A quantidade adsorvida de corante foi utilizada como variável resposta nessa otimização.

Tabela 1: Planejamento de Experimentos para avaliação da faixa ótima de $\mathrm{pH}$.

\begin{tabular}{cccc}
\hline ORDEM & PONTO & $\mathrm{pH}$ & $\mathrm{Co}(\mu \mathrm{mol} / \mathrm{L})$ \\
\hline 1 & axial & 6 & 138,6 \\
2 & axial & 6 & 11,4 \\
3 & fatorial & 3 & 120 \\
4 & axial & 10,2 & 75 \\
5 & axial & 1,8 & 75 \\
6 & central & 6 & 75 \\
7 & central & 6 & 75 \\
8 & central & 6 & 75 \\
9 & fatorial & 3 & 30 \\
10 & fatorial & 9 & 30 \\
11 & fatorial & 9 & 120 \\
12 & central & 6 & 75 \\
\hline
\end{tabular}

\subsection{Ensaios cinéticos de adsorção}

Os ensaios cinéticos foram realizados em $\mathrm{pH}$, previamente avaliado como o melhor meio para o processo, e sob condições próximas da ambiente (temperatura de $30{ }^{\circ} \mathrm{C}$ ), seguindo a metodologia da imersão em banho finito, conforme descrita em Rouquerol, Rouquerol e Sing (1999).

Todos estes ensaios cinéticos foram conduzidos em células experimentais constituídas por frascos erlenmeyers de borosilicato de $125 \mathrm{~mL}$, agitados a 160 rpm em incubadora com agitação orbital (Marconi MA 410/CF). Esse valor de agitação era o máximo fornecido pelo equipamento e foi utilizado de modo a minimizar o tempo de equilíbrio do processo.

Previamente ao contato da solução corante com o adsorvente, $20 \mathrm{~mL}$ de água destilada e aproximadamente $0,2 \mathrm{~g}$ do adsorvente eram deixados em contato na célula por 24 horas para saturação do adsorvente com o solvente. Somente após esse tempo de saturação eram adicionados $20 \mathrm{~mL}$ da solução corante à célula e iniciavam-se as corridas experimentais cinéticas, as quais duravam 48 horas. Nestes testes tanto a SGM como a sílica gel não funcionalizada foram utilizados como adsorventes nos ensaios.

Nestes ensaios, as soluções de corante adicionadas tinham concentrações de $100 \mathrm{mg} / \mathrm{L}$ e de $200 \mathrm{mg} / \mathrm{L}$. Assim, as soluções de corante nas células cinéticas poderiam ser assumidas com concentrações iniciais de 50 e de $100 \mathrm{mg} / \mathrm{L}$, respectivamente. Estes valores de concentração foram selecionados por motivos analíticos, haja vista que a concentração de $100 \mathrm{mg} / \mathrm{L}$ era o limite superior da curva de calibração do método de quantificação 
da solução corante, e considerando os resultados obtidos na otimização do $\mathrm{pH}$ dos ensaios.

Durante o tempo da corrida cinética, em tempos prédefinidos, alíquotas de $7 \mathrm{~mL}$ eram retiradas das células, tamponadas para $\mathrm{pH} 7,0$, centrifugadas e analisadas em espectrofotômetro UV/VIS.

Os dados cinéticos obtidos foram usados no ajuste dos modelos de pseudo-primeira e pseudo-segunda ordem expressos na Equação 1 e na Equação 2, respectivamente.

$$
\begin{aligned}
& \frac{d q_{t}}{d t}=k_{1}\left(m_{1}-q_{2}\right) \\
& \frac{d q_{t}}{d t}=k_{2}\left(m_{2}-q_{t}\right)^{2}
\end{aligned}
$$

em que $q_{t}$ é a quantidade adsorvida num instante $t$, $k_{1}$ e $m_{1}$, e $k_{2}$ e $m_{2}$ são os parâmetros dos modelos. Os coeficientes de determinação e os desvios relativos médios para os ajustes também foram calculados.

\subsection{Ensaios de equilíbrio de adsorção}

Os ensaios de equilíbrio também foram realizados em pH 3 e temperatura de $30^{\circ} \mathrm{C}$, conduzidos nas mesmas células utilizadas no ensaio de cinética, seguindo a metodologia da imersão em banho finito. A agitação utilizada também foi de $160 \mathrm{rpm}$.

Alíquotas de $20 \mathrm{~mL}$ de soluções de corante também foram adicionadas aos frascos erlenmeyers, que já continham $0,2 \mathrm{~g}$ do adsorvente previamente saturado com $20 \mathrm{~mL}$ de água destilada por 24. As concentrações iniciais do adsortivo nas células de equilíbrio variaram de 5 a $600 \mathrm{mg} / \mathrm{L}$. Escolheu-se o valor inferior dessa faixa ( $5 \mathrm{mg} / \mathrm{L}$ ) como o dobro do valor do limite de quantificação do método analítico utilizado, enquanto que a escolha do valor superior $(600 \mathrm{mg} / \mathrm{L})$ pretendia verificar a possível tendência de saturação do adsorvente em altas concentrações.

Os erlenmeyers foram agitados no mesmo equipamento e nas mesmas condições dos ensaios cinéticos. A solução ficou em contato com o adsorvente por 1 hora de modo a garantir o equilíbrio, cujo tempo foi estimado a partir dos ensaios cinéticos. As alíquotas eram então retiradas das células, tamponadas em pH 7,0, centrifugadas, e analisadas espectrofotometricamente.

Os dados de equilíbrio determinados foram utilizados no ajuste das isotermas de Langmuir e de Freundlich, representadas na Equação 3 e na Equação 4, respectivamente.

$$
q_{e}=\frac{m_{L} k_{L} C_{e}}{1+k_{L} C_{e}}
$$

$$
q_{e}=k_{F} C_{e}^{1 / n}
$$

em que $q_{e}$ é a quantidade adsorvida no equilíbrio, $m_{L}$ e $k_{L}$, e $k_{F}$ e $n$ são os parâmetros dos modelos. Os coeficientes de determinação e os desvios relativos médios para os ajustes também foram calculados.

\section{RESULTADOS E DISCUSSÃO}

\subsection{Ensaios preliminares de caracterização do ad- sorvente e de otimização do pH dos ensaios}

Inicialmente, a modificação do adsorvente foi confirmada pela comparação dos espectrogramas FTIR obtidos nas mesmas condições para a sílica gel comercial antes e depois do procedimento de funcionalização (silanização), os quais estão apresentados na Figura 3

Nestes espectrogramas, observam-se as diminuições das bandas características dos grupos silanóis e siloxanos $\left(3448 \mathrm{~cm}^{-1}, 1085 \mathrm{~cm}^{-1}, 954 \mathrm{~cm}^{-1}, 799 \mathrm{~cm}^{-1}\right)$, juntamente com o surgimento de bandas características muito discretas de estiramento $=\mathrm{CH} 2$ entre 2850 e $2950 \mathrm{~cm}^{-1}$. Espectrogramas semelhantes e as mesmas variações espectrais foram observadas por Donia et al. (2009) quando do desenvolvimento desse procedimento de silanização da silica gel, indicando que a modificação proposta foi obtida.

A curva obtida nos ensaios de PCZ, mostrada na Figura 4 indicaram uma mudança na carga superficial do adsorvente avaliado em pH 6,7 (valor em que ela cruza a linha de $45^{\circ}$ ). Isto é, o adsorvente tem sua carga superficial positiva em solução aquosa para valores de $\mathrm{pH}$ inferiores a 6,7, o que deve favorecer a adsorção do corante aniônico estudado nessa faixa de $\mathrm{pH}$.

Complementarmente, a superfície de resposta obtida nos ensaios de otimização do $\mathrm{pH}$ por DCCR para o processo adsortivo avaliado, apresentada no diagrama de contorno da Figura 5 também indica que as capacidades de adsorção do corante no adsorvente são incrementadas à medida que o $\mathrm{pH}$ do meio vai se tornando mais ácido, principalmente quando os valores são inferiores a 4,0 (maiores desejabilidades).

Estes resultados sugerem que o mecanismo de adsorção pode ser explicado pela protonação dos grupos amino na superfície modificada da sílica gel, os quais interagem com os ânions sulfônicos do corante por adsorção eletrostática, conforme foi observado por Zhang et al. (2015).

Ainda, considerando tais resultados, o pH 3 (nível inferior de $\mathrm{pH}$ do planejamento experimental) foi selecionado para os experimentos cinéticos e de equilíbrio da adsorção do corante na SGM. 


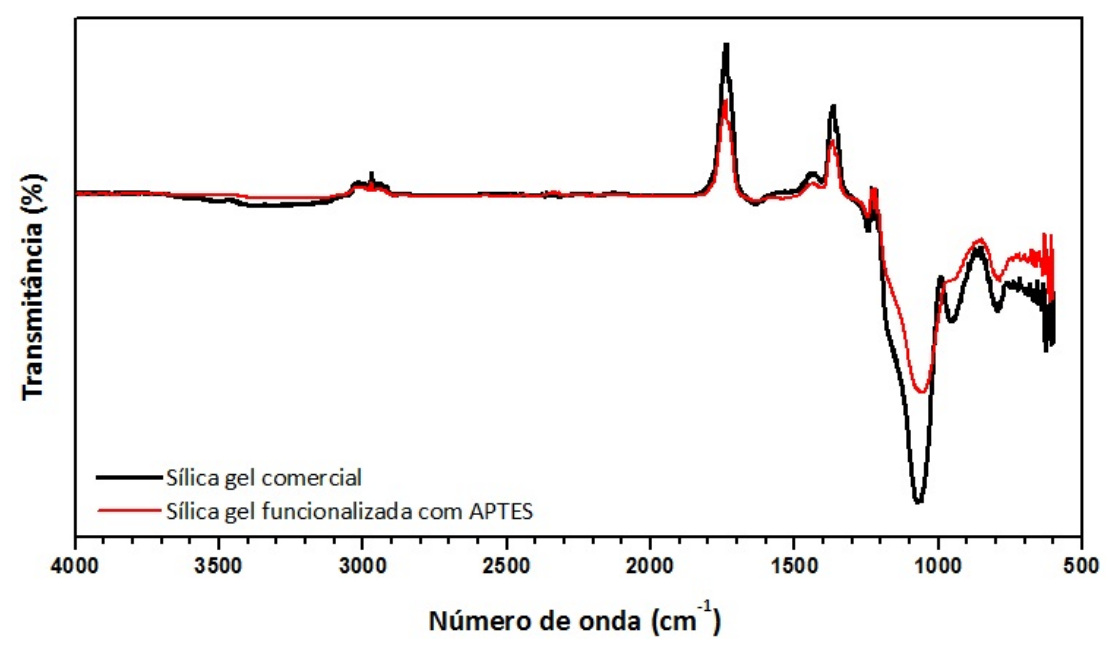

Figura 3: Os espectros de FTIR da sílica gel comercial e sílica gel modificada.

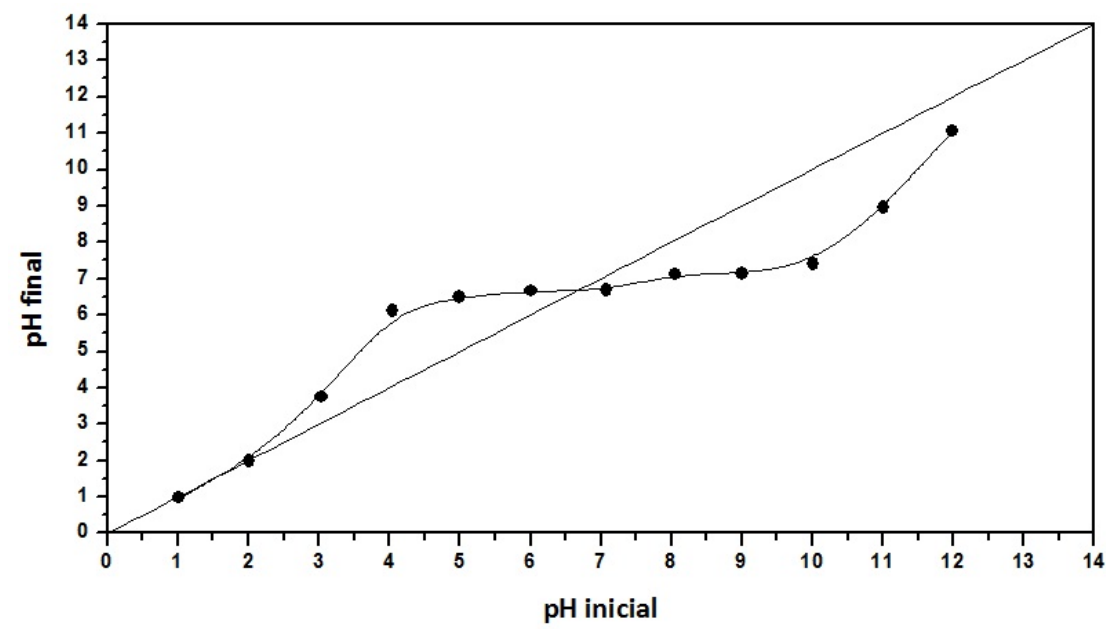

Figura 4: Curva PCZ obtida para a sílica gel modificada. 


\begin{abstract}
Ademais, pode ser observado no diagrama, que as quantidades adsorvidas aumentam à medida que a concentração inicial do adsortivo aumenta, indicando que na faixa de concentrações avaliadas nesse teste o adsorvente ainda não foi saturado com o corante, podendo-se trabalhar com concentrações mais elevadas nos testes adsortivos.
\end{abstract}

\subsection{Ensaios cinéticos e de equilíbrio de adsorção}

Os ensaios cinéticos e de equilíbrio foram, então, conduzidos seguindo a metodologia já descrita, fornecendo os resultados apresentados nas Figuras 6 e 7, respectivamente, para a sílica gel modificada. Os modelos cinéticos e de isotermas ajustados aos dados também estão apresentados nestas figuras. Mencione-se que os ensaios cinéticos realizados com a sílica não funcionalizada (modificada) mostraram que este material praticamente não adsorve o corante no pH e nas concentrações iniciais testadas, corroborando a importância da protonação dos grupos aminos na adsorção do corante azoico avaliado.

Ressalte-se que os parâmetros estimados no ajuste dos modelos cinéticos e de equilíbrio estão dispostos na Tabela 2 e na Tabela 3, respectivamente. Os coeficientes de determinação $\left(R^{2}\right)$ e a média dos erros relativos $\left(\delta_{r}\right)$ obtidos no ajuste dos modelos aos dados experimentais também foram calculados e dispostos nestas duas tabelas.

A partir da Figura 6é possível verificar que a cinética do processo é muito rápida, considerando que em menos de 5 minutos as quantidades adsorvidas já chegam a mais de $95 \%$ da quantidade adsorvida no equilíbrio. Ademais, pode-se verificar que as quantidades adsorvidas praticamente não variam mais em tempos de 30 a 40 minutos de contato, estimando-se o tempo de equilíbrio em 40 minutos.

Tempos de equilíbrio similares foram encontrados por Wang et al. (1998) e Zhang et al. (2015) para o mesmo corante em outros adsorventes (Lodo particulado e nanopartículas de $\mathrm{Fe} 3 \mathrm{O} 4$ modificadas) e foram relativamente inferiores ao tempo de equilíbrio (3 horas) observado por Pham, Kobayashi e Adachi (2015) utilizando $\alpha$-alumina. É importante destacar que uma cinética rápida favorece a aplicação do processo em escala industrial.

Os resultados ainda revelam que dentre os modelos cinéticos simplificados testados, o de pseudo-segunda ordem representou melhor os dados experimentais cinéticos obtidos (erros médios de até 6,5\%), sugerindo que este modelo pode ser usado na estimativa de parâmetros de projeto e de operação de unidades de adsorção em batelada para o corante em fase aquosa.
Para os dados de equilíbrio, ambos os modelos típicos de isotermas usados se mostraram pouco eficientes na estimativa desses dados, haja vista que os desvios em relação aos dados experimentais foram superiores a $30 \%$, em média, devido principalmente às estimativas em baixas concentrações. Contudo, a estimativa da quantidade máxima adsorvida obtida a partir do modelo de Langmuir pode ser comparada com aquela determinada para outros adsorventes testados na literatura.

O valor de capacidade máxima para a adsorção do Ponceau 4R estimado nesse estudo $(47,218 \mathrm{mg} / \mathrm{g})$ é consideravelmente superior aos valores determinados por Pham, Kobayashi e Adachi (2015) em $\alpha$-alumina $(1,0 \mathrm{mg} / \mathrm{g})$ e por Weng (2002) em cinzas de lodo de esgoto doméstico (3,25 a 5,70 mg/g), bem como é comparável às capacidades obtidas por Wang et al. (1998) para lodo particulado $(84,6 \mathrm{mg} / \mathrm{g})$, por Weng et al. (2010) usando nanopartículas magnéticas modificadas de $\mathrm{Fe}_{3} \mathrm{O}_{4}(67 \mathrm{mg} / \mathrm{g})$ e por Suresh, Sugumar e Maiyalagan (2011) ao usar carvão ativado produzido a partir de sementes da árvore do curry $(53,19 \mathrm{mg} / \mathrm{g})$.

Alguns resultados muito satisfatórios e que corroboram a efetividade dos grupos amino protonados na adsorção de corantes azoicos sulfonados, particularmente o Ponceau 4R, foram obtidos por Li e Lei (2012), com um copolímero reticulado de poliaminoamida e ciclodextrina, e por Zhang et al. (2015) com nanopartículas magnéticas de $\mathrm{Fe}_{3} \mathrm{O}_{4}$ modificadas com poli-lisina, cujas capacidades máximas de adsorção estimadas foram de $266,8 \mathrm{mg} / \mathrm{g}$ e de $245 \mathrm{mg} / \mathrm{g}$, respectivamente. Estes autores atribuem tais capacidades ao maior número de grupos amino na superfície adsorvente e à correspondente adsorção eletrostática em pH ácido.

\section{CONCLUSÕES}

A sílica gel organofuncionalizada com APTES se mostra um adsorvente promissor na remoção de corantes azoicos como o Ponceau 4R. O processo adsortivo deve ser conduzido em pH ácido, considerando que uma adsorção eletrostática ocorrerá, decorrente da interação entre os grupos amino protonados da superfície da SGM e os grupos sulfônicos do Ponceau 4R, conforme indicaram os resultados desse estudo e os dados da literatura. Uma capacidade máxima de 47,218 mg/g e uma cinética muito rápida (tempo de equilíbrio de 40 minutos) para a adsorção do corante estudado na SGM foram observadas nas condições experimentais testadas. O processo pode ser bem representado por um modelo cinético de pseudo-segunda ordem, mas as isotermas de equilíbrio de Langmuir e de Freundlich não se ajustaram satisfatoriamente aos dados experimentais determinados, devendo-se buscar outros modelos para a des- 


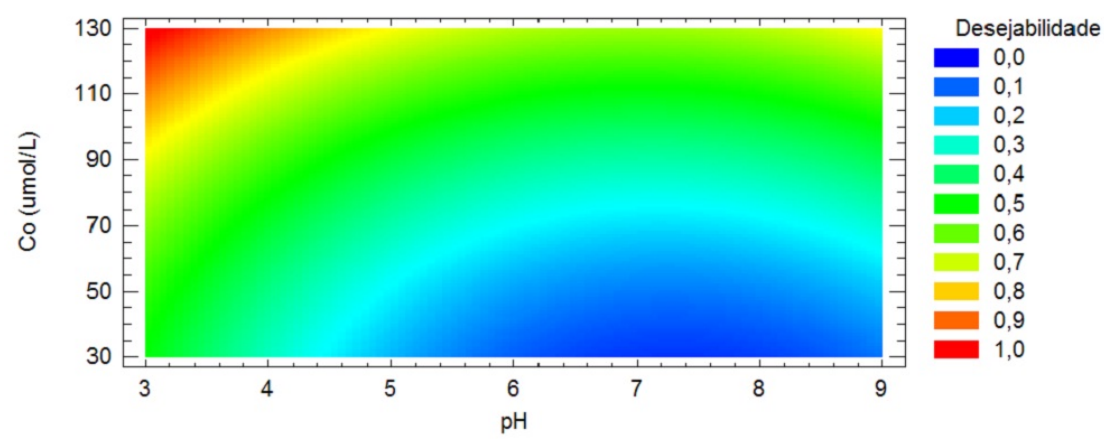

Figura 5: Superfície de resposta a partir dos experimentos realizados segundo o DCCR, maximizando a quantidade adsorvida do corante Ponceau 4R.

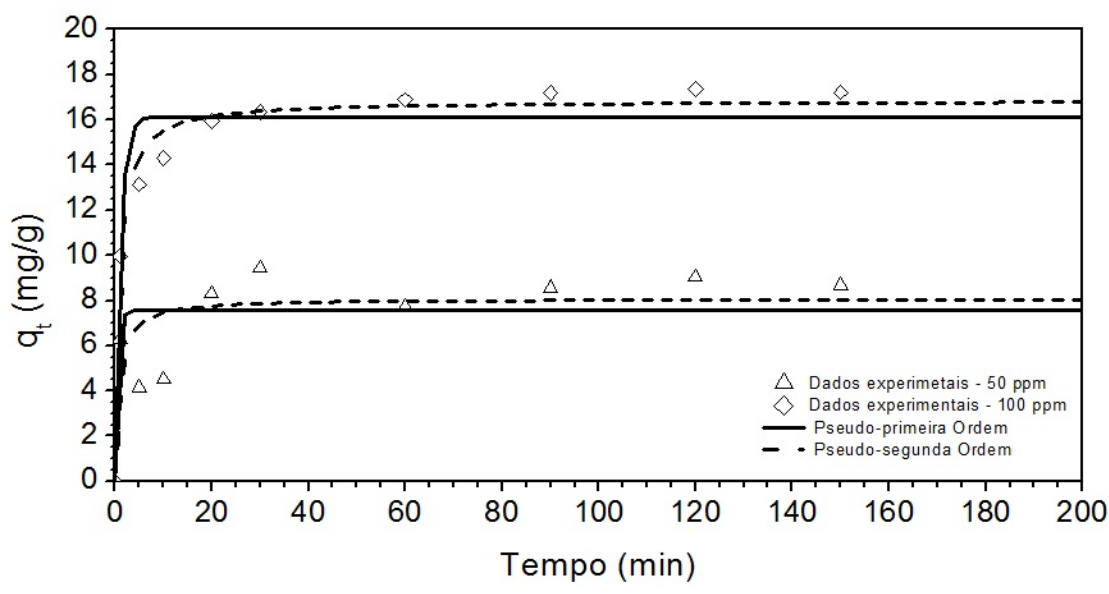

Figura 6: Curvas cinéticas para a adsorção do corante Ponceau 4R na sílica gel funcionalizada.

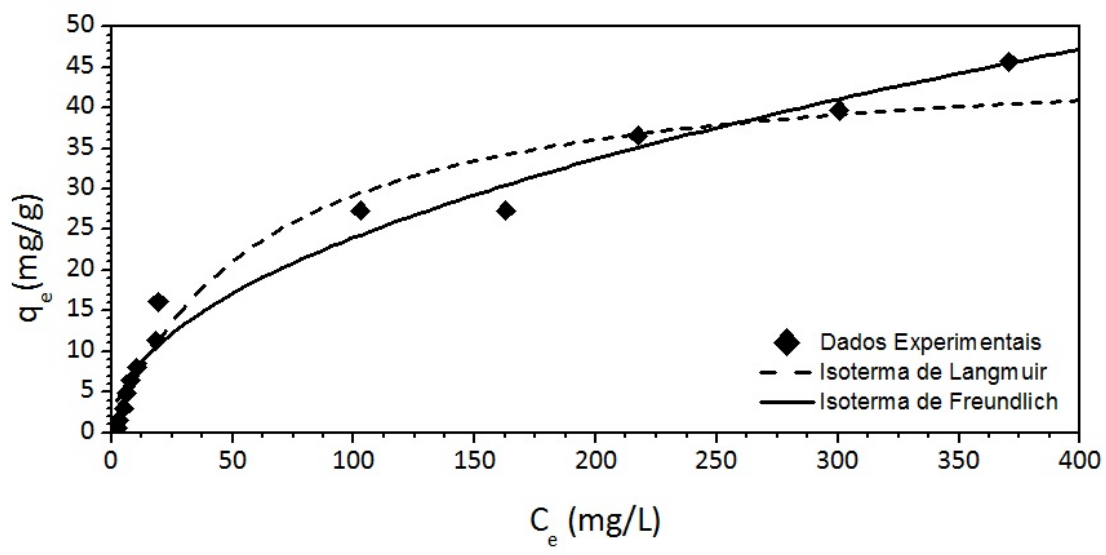

Figura 7: Isotermas de equilíbrio para a adsorção do corante na sílica gel funcionalizada.

Conex. Ci. e Tecnol. Fortaleza/CE, v. 10, n. 2, p. 7 - 16, jul. 2016 

APTES

Tabela 2: Parâmetros dos modelos cinéticos ajustados na remoção do Ponceau 4R sobre a sílica gel funcionalizada com APTES a $30{ }^{\circ} \mathrm{C}$. Concentrações iniciais $\left(\mathrm{C}_{0}\right)$ do corante iguais a 50 e $100 \mathrm{mg} / \mathrm{L}$.

\begin{tabular}{|c|c|c|c|c|c|c|c|c|}
\hline \multirow{2}{*}{$\begin{array}{c}\mathrm{C}_{0} \\
(\mathrm{mg} / \mathrm{L})\end{array}$} & \multicolumn{4}{|c|}{ Pseudo-primeira ordem } & \multicolumn{4}{|c|}{ Pseudo-segunda ordem } \\
\hline & $\begin{array}{c}\mathrm{m}_{1} \\
(\mathrm{mg} / \mathrm{g})\end{array}$ & $\begin{array}{c}\mathrm{k}_{1} \\
\left(\min ^{-1}\right)\end{array}$ & $\mathrm{R}_{2}$ & $\delta_{r}$ & $\begin{array}{c}\mathrm{m}_{2} \\
(\mathrm{mg} / \mathrm{g})\end{array}$ & $\begin{array}{c}\mathrm{k}_{2} \\
\mathrm{~g}(\mathrm{mg} \cdot \mathrm{min})^{-1}\end{array}$ & $\mathrm{R}_{2}$ & $\delta_{r}$ \\
\hline 50 & 7,5462 & 1,7748 & 7 & ט & 8,0808 & 0,1434 & 0,6 & $6,5 \%$ \\
\hline 100 & 16,0989 & 0,9072 & 0,5852 & $21,5 \%$ & 16,8449 & 0,0686 & 0,9793 & $4,0 \%$ \\
\hline
\end{tabular}

Tabela 3: Parâmetros dos modelos ajustados das isotermas de Langmuir e de Freundlich na remoção do Ponceau 4R sobre a sílica gel funcionalizada com APTES a $30^{\circ} \mathrm{C}$.

\begin{tabular}{cccc|cccc}
\hline \multicolumn{3}{c|}{ Isoterma de Langmuir } & \multicolumn{4}{c}{ Isoterma de Freundlich } \\
\hline $\mathrm{m}_{L}$ & $\mathrm{~K}_{L}$ & $\mathrm{R}^{2}$ & $\delta_{r}$ & $\mathrm{~K}_{F}$ & $n$ & $\mathrm{R}^{2}$ & $\delta_{r}$ \\
$(\mathrm{mg} / \mathrm{g})$ & $(\mathrm{L} / \mathrm{g})$ & & & $(\mathrm{L} / \mathrm{g})$ & & & \\
47,2180 & 0,0162 & 0,9608 & $31,1 \%$ & 2,5435 & 2,0506 & 0,9717 & $76,8 \%$ \\
\hline
\end{tabular}

crição do processo. Estudos complementares em diferentes condições operacionais (e.g., temperatura, força iônica) e com outros corantes azoicos devem ser conduzidos de modo a confirmar a eficiência do adsorvente na remoção de corantes azoicos sulfonados.

\section{AGRADECIMENTOS}

Os autores agradecem à Fundação Cearense de Apoio ao Desenvolvimento Científico e Tecnológico (FUNCAP), e ao Instituto Federal de Educação, Ciência e Tecnologia do Ceará (IFCE), pelas bolsas de mestrado, de iniciação cientifica e de iniciação científica junior concedidas ao nosso grupo de pesquisa e ao Laboratório de Processos Químicos e Ambientais e ao Laboratório de Tecnologia Química do Departamento de Química e Meio Ambiente do IFCE - Campus Fortaleza pelo suporte analítico e financeiro.

\section{REFERÊNCIAS}

ABRAMIAN, L.; EL-RASSY, H. Adsorption kinetics and thermodynamics of azo-dye orange ii onto highly porous titania aerogel. Desalination, v. 278, p. 412-423, 2011.

AHMAD, A. A.; HAMEED, B. H. Fixed-bed adsorption of reactive azo dye onto granular activated carbon prepared from waste. Journal of Hazardous Materials, v. 175, p. 298-303, 2010.

ANDRZEJEWSKA, A.; KRYSZTAFKIEWICZ, A.; JESIONOWSKI, T. Treatment of textile dye wastewater using modified silica. Dyes and Pigments, v. 75, p. 116-124, 2007.

BOUCHERIT, N.; ABOUSEOUD, M.; ADOUR, L. Degradation of direct azo dye by cucurbita pepo free and immobilized peroxidase. Journal of Environmental Sciences, v. 25, p. 1235-1244, 2013.

CESTARI, A. R.; VIEIRA, E. F. S.; VIEIRA G. S.AND COSTA, L. P.; TAVARES, A. M. G.; LOH W., A. C. The removal of reactive dyes from aqueous solutions using chemically modified mesoporous silica in the presence of anionic surfactant - the temperature dependence and a thermodynamic multivariate analysis. Journal of Hazardous Materials, v. 161, p. 307-316, 2009.

DONIA, A. M.; ATIA, A. A.; AL-AMRANI, W. A.; EL-NAHAS, A. M. Effect of structural properties of acid dyes on their adsorption behaviour from aqueous solutions by amine modified silica. Journal of Hazardous Materials, v. 161, p. 1544-1550, 2009.

FAHMI ARIFFIN, A.; ARSHAD, S. M.; ABIDIN, C. Z. A.; RAHMAT, N. R. Decolourization and cod removal of azo dye solution by repeated ozonation and biodegradation. In: BABY, S.; SANDHU, P. S. (Ed.). International Conference on Environmental Science and Technology. Bangkok, Thailand: Research Publishing Services, 2010. p. 149-153.

FRANCISCON, E.; GROSSMAN, M. J.; PASCHOAL, J. A. R.; REYES, F. G. R.; DURRANT, L. R. Decolorization and biodegradation of reactive sulfonated azo dyes by a newly isolated brevibacterium sp. strain vn-15. SpringerPlus, v. 1, n. 37, p. 1-10, 2012.

GUILARDUCI, V. V. S.; MESQUITA, J. P.; MARTELLI, P. B.; GORGULHO, H. F. Adsorção de fenol sobre carvão ativado em meio alcalino. Química Nova, v. 29, p. 1226-1232, 2006. 
ADSORÇÃO EM MEIO AQUOSO DO CORANTE AZO PONCEAU 4R SOBRE SÍLICA GEL ORGANOFUNCIONALIZADA COM APTES

GUPTA, V. K.; GUPTA, B.; RASTOGI, A.; AGARWAL, S.; NAYAK, A. A comparative investigation on adsorption performances of mesoporous activated carbon prepared from waste rubber tire and activated carbon for a hazardous azo dye - acid blue 113. Journal of Hazardous Materials, v. 186, p. 891-901, 2011.

GUPTA, V. K.; SUHAS. Application of low-cost adsorbents for dye removal - a review. Journal of Environmental Management, v. 90, p. 2313-2342, 2009.

KODAM, K. M.; GAWAI, K. R. Decolorisation of reactive red 11 and 152 azo dyes under aerobic conditions. Indian Journal of Biotechnology, v. 5, p. 422-424, 2006.

KRYSZTAFKIEWICZ, A.; BINKOWSKI, S.; JESIONOWSKI, T. Adsorption of dyes on a silica surface. Applied Surface Science, v. 199, p. 31-39, 2002.

KUSHWAHA, A. K.; GUPTA, N.; CHATTOPADHYAYA, M. C. Enhanced adsorption of malachite green dye on chemically modified silica gel. Journal of Chemical and Pharmaceutical Research, v. 2, p. 34-45, 2010.

LI, N.; LEI, X.-M. Adsorption of ponceau 4r from aqueous solutions by polyamidoamine-cyclodextrin crosslinked copolymer. Journal of Inclusion Phenomena and Macrocyclic Chemistry, v. 74, p. 167-176, 2012.

MAHMOODI, N. M.; KHORRAMFAR, S.; NAJAFI, F. Amine-functionalized silica nanoparticle: Preparation, characterization and anionic dye removal ability. Desalination, v. 279, p. 61-68, 2011.

MAHMOODI, N. M.; SDEGHI, U.; MALEKI, A.; HAYATI, B.; NAJAFI, F. Synthesis of cationic polymeric adsorbent and dye removal isotherm, kinetic and thermodynamic. Journal of Industrial and Engineering Chemistry, v. 20, p. 2745-2753, 2014.

MALIK, R.; RAMTEKE, D. S.; WATE, S. R. Adsorption of malachite green on groundnut shell waste based powdered activated carbon. Waste Management, v. 27, p. 1129-1138, 2007.

MITTAL, A.; MITTAL, J.; MALVIYA, A.; GUPTA, V. Adsorptive removal of hazardous anionic dye "congo red"from wastewater using waste materials and recovery by desorption. Journal of Colloid and Interface Science, v. 340, p. 16-26, 2009.
NAM, S.; RENGANATHAN, V.; TRATNYEK, P. G. Substituent effects on azo dye oxidation by the feiii-edta-h2o2 system. Chemosphere, v. 45, p. 59-65, 2001.

PHAM, T. D.; KOBAYASHI, M.; ADACHI, Y. Adsorption characteristics of anionic azo dye onto large a-alumina beads. Colloid and Polymer Science, v. 293, p. 1877-1886, 2015.

RODRIGUES, M. I.; EMMA, A. F. Planejamento de experimentos e otimização de processos. 1. ed. Campinas: Casa do Pão Editora, 2005.

ROUQUEROL, F.; ROUQUEROL, J.; SING, K. Adsorption by Powders \& Porous Solids: principles, methodology and applications. San Diego: Academic Press, 1999. 467p.

ROY, A.; ADHIKARI, B.; MAJUMDER, S. B. Equilibrium, kinetic, and thermodynamic studies of azo dye adsorption from aqueous solution by chemically modified lignocellulosic jute fiber. Industrial \& Engineering Chemistry Research, v. 52, p. 6502-6512, 2013.

SHARMA, P.; KAUR, H.; SHARMA, M.; SAHORE, $\mathrm{V}$. A review on applicability of naturally available adsorbents for the removal of hazardous dyes from aqueous waste. Environmental Monitoring and Assessment, v. 183, p. 151-195, 2011.

SHIRMARDI, M.; MESDAGHINIA, A.; MAHVI, A. H.; NASSERI, S.; NABIZADEH, R. Kinetics and equilibrium studies on adsorption of acid red 18 (azo-dye) using multiwall carbon nanotubes (mwcnts) from aqueous solution. E-Journal of Chemistry, v. 9, p. 2371-2383, 2012.

SURESH, S.; SUGUMAR, R. W.; MAIYALAGAN, T. Adsorption of acid red 18 from aqueous solution onto activated carbon prepared from murraya koenigii (curry tree) seeds. Asian Journal of Chemistry, v. 23, p. 219-224, 2011.

WANG, J.; HUANG, C. P.; ALLEN, H. E.; CHA, D. K.; KIM, D.-W. Adsorption characteristics of dye onto sludge particulates. Journal of Colloid and Interface Science, v. 208, p. 518-528, 1998.

WANG, L. Application of activated carbon derived from "waste"bamboo culms for the adsorption of azo disperse dye: Kinetic, equilibrium and thermodynamic studies. Journal of Environmental Management, v. 102, p. 79-87, 2012. 
WENG, C.-H. Adsorption characteristics of new coccine dye on to sludge ash. Adsorption Science \& Technology, v. 20, p. 669-681, 2002.

WENG, C.-H.; LIN, Y.-T.; YEH, C.-L.; SHARMA, Y. C. Magnetic fe3o4 nanoparticles for adsorptive removal of acid dye (new coccine) from aqueous solutions. Water Science and Technology, v. 62, p. 844-851, 2010.

XIA, C. et al. Adsorption properties of congo red from aqueous solution on modified hectorite: kinetic and thermodynamic studies. Desalination, v. 265, p. 81-87, 2011 .

YIGITOGLU, M.; TEMOCIN, Z. Removal of benzidine-based azo dye from aqueous solution using amide and amine-functionalized poly(ethylene terephthalate) fibers. Fibers and Polymers, v. 11, p. 996-1002, 2010.

ZHANG, Q. et al. Deciphering effects of chemical structure on azo dye decolorization/degradation characteristics: Bacterial vs. photocatalytic method. Journal of the Taiwan Institute of Chemical Engineers, v. 43, p. 760-766, 2012.

ZHANG, Y.-R.; SU, P.; HUANG, J.; WANG, Q.-R.; ZHAO, B.-X. A magnetic nanomaterial modified with poly-lysine for efficient removal of anionic dyes from water. Chemical Engineering Journal, v. 262, p. 313-318, 2015. 